\title{
Synthesis of DSPDA for second order nonlinear optical materials
}

\author{
li Shen, wen bing Yang, ning ren Jin, miao lan Shen \\ Shaoxing University Yuanpei College, Zhejiang University of Technology, Shaoxing 312000, \\ Zhejiang
}

Keywords:Second order nonlinear optical materials, group- $E$-diphenylethene compounds, $N, N^{\prime}$-diacetyl-2-(2,4- dimethoxystyryl)-5-nitro -1,4-phenylenediamine (DSPDA)

\begin{abstract}
Second order nonlinear optical materials have been in a very important position because of its special excellent optical properties, The large nonlinear optical coefficient chromophore molecule is the key to the excellent nonlinear optical materials. In this study, the industrialized synthetic process of multi-substituted group- $E$-diphenylethene compounds with push-pull electronic structure was studied, based on the properties of diphenylethene compounds and the advantage of diphenylethene compounds which have the push-pull electronic system. The chromophore compounds $N, N^{\prime}$-diacetyl-2-(2,4-dimethoxystyryl)-5-nitro-1,4-phenylenediamine (DSPDA), were synthesized and optimized to adjust the industrial production.
\end{abstract}

\section{Introduction}

Second-order nonlinear optical material has a series of advantages,such as high optical coefficient, low optical loss, short response time and so on, it has been widely used in optical communication, optical information processing and other fields ${ }^{[1]}$. Excellent second order nonlinear optical materials is essential to have high optical coefficient chromophores and asymmetric center ${ }^{[2]}$, In this paper, the synthetic process of multi-substituted group- $E$ - diphenylethene compounds was studied, based on the chromophores molecules of diphenylethenea compounds with push-pull electronic system.

\section{The present status of synthesizing group-E-diphenylethene compounds both inside and outside China}

The main reason plague group-E-diphenylethene compounds extensive application is how to carry out the current situation of group- $E$-diphenylethene compounds industrial production. The main methods of synthesis group- $E$ - diphenylethene compounds as follow:

Witting reaction: witting reaction is nucleophilic addition reaction, witting reagent and carbonyl group of aldehydes andketones reacted to olefins. nucleophilic triphenylphosphine and halogenated hydrocarbon as raw materia, reacted to get quaternary phosphonium salts and then used strong alkali to remove $\alpha$-hydrogen atom in alkyl, finally get witting reagent, as shown in figure 1:

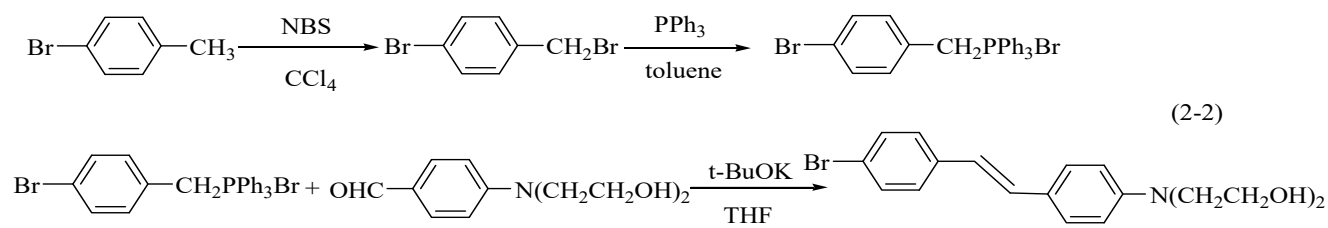

Figure 1. Witing reaction synthesis route

The stilbenes compounds obtained from the synthetic route not only had big yield but also had high purity, but the reaction steps was complex, this synthetic route cannot complete one pot 
reaction, each step must operated separately, reaction materials, reagents and catalysts were expensive, the cost was high, so this method generally applicable in the laboratory.

W. J. FARRISSEY. Et al used two-step method to synthesize group- $E$-diphenylethene compounds, the method was as follows:

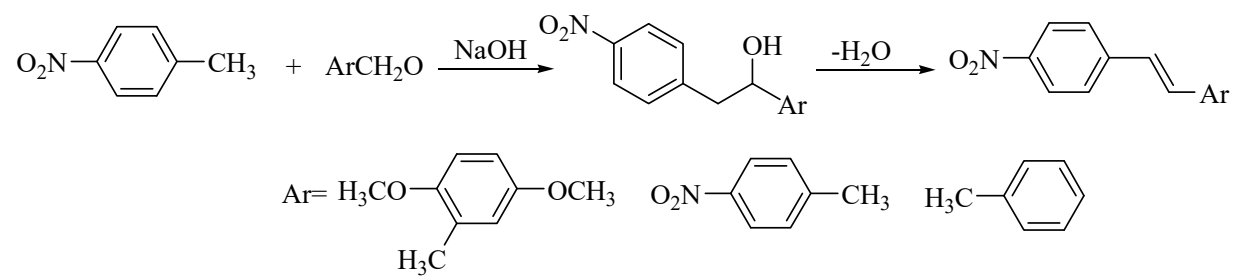

Figure 2. two-step method synthetic route

The method used $\mathrm{NaOH}$ as catalyst to synthesize stilbenes compounds. It was relatively simple, but product purity was low, it was not suitable for the synthesis of multi-substituted group- $E$-diphenylethene compounds with push-pull electronic structure.

Using polyethylene glycol as a catalyst to synthesize stilbenes compounds directly ${ }^{[5]}$, As shown in figure3, Although the method was simple, but the purity of target product was very low.

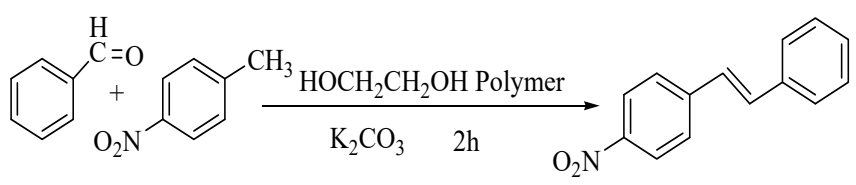

Figure 3. polyethylene glycol synthesis

\section{Research on new methods of synthesizing group-E-diphenylethene compounds and process optimization}

A new method of synthesizing group- $E$-diphenylethene compounds and qualitative analysis. This article studies the innovation of the reaction is based on the way of synthetic group- $E$-diphenylethene compounds which is developed by Witting and Weder Et al. According to the double bond of carbon to oxygen, polarization make carbon atoms has a part positive charge, which can accept attack of nucleophilic particle, thus produce become new addition product, synthetic route is as follows.

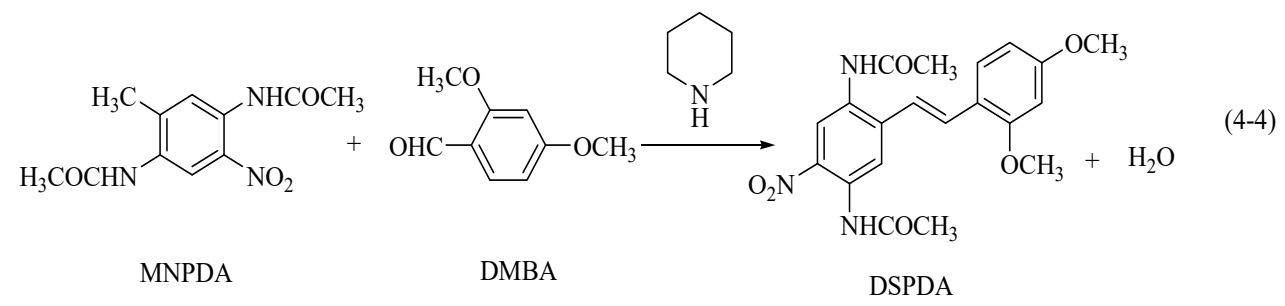

Figure 4. DSPDA synthetic route

This reaction synthesize new group- $E$-diphenylethene monomer(DSPDA), piperidine was used as catalyst to capture hydrogen, DMSO as solvent for reaction, the target products obtained are as follows: 


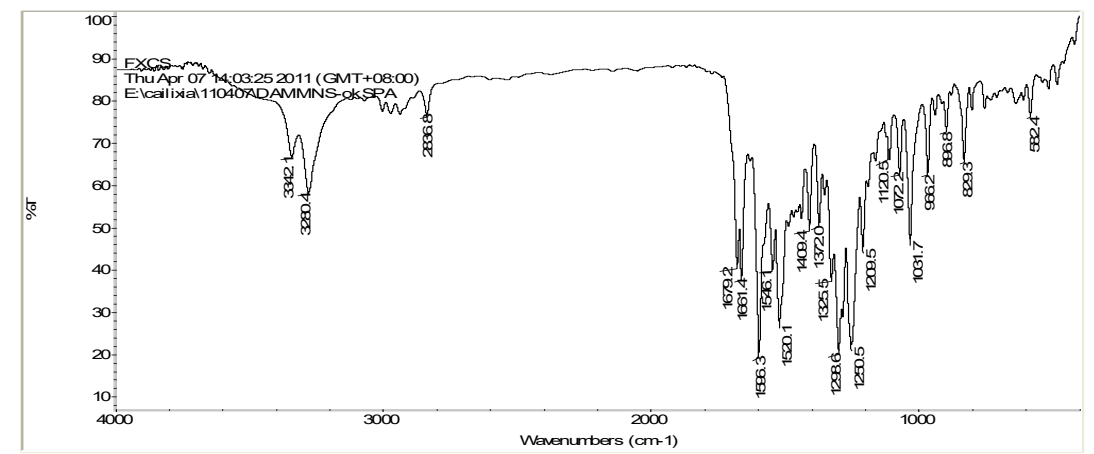

Figure 5. DSPDA infrared spectrogram

Table 1. DSPDA infrared spectrogram analysis tables

\begin{tabular}{lccc}
\hline group & Characteristic spectrum peak $\mathrm{cm}^{-1}$ & group & \multicolumn{2}{c}{ Characteristic spectrum peak $\mathrm{cm}^{-1}$} \\
\hline$=\mathrm{CH}$ & 966.2 & amide II & 1546.1 \\
$\mathrm{CH}=\mathrm{CH}$ & 1679.2 & amideIII & 1325.5 \\
$\mathrm{NH}$ & 3342.13280 .4 & benzene ring & 1596.31546 .1 \\
amide I C=O & 1661.4 & $\mathrm{CH}_{3}$ & 2836.8 \\
\hline
\end{tabular}

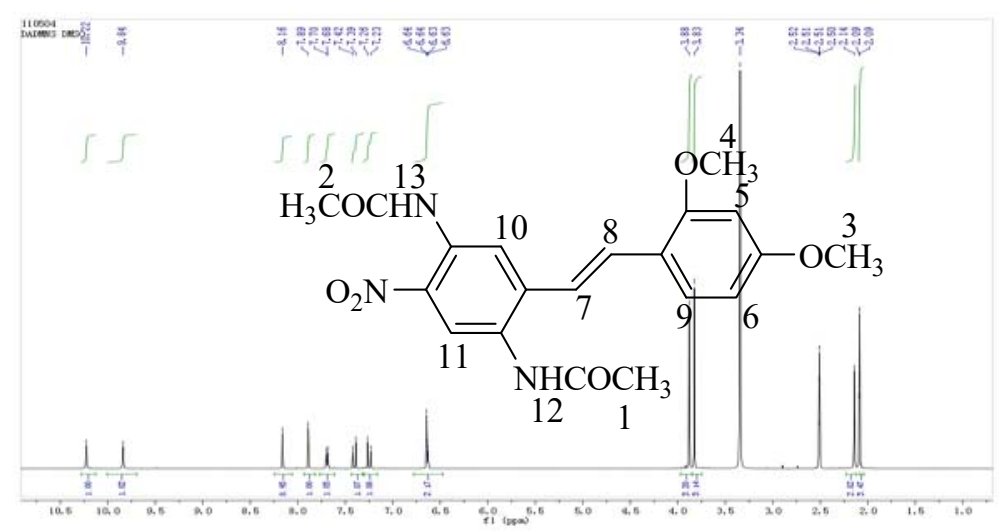

Figure 6. HNMR of DSPDA

Table 2. HNMR of DSPDA analysis table

\begin{tabular}{|c|c|c|c|c|c|c|c|c|c|}
\hline \multicolumn{3}{|c|}{ Position $\delta_{H}\left(10^{-6}\right)$} & Position & & 6) $\mathrm{H}$ & Position & $\delta_{H}\left(10^{-6}\right)$ & $\mathrm{H}$ & Position $\quad \delta_{\mathrm{H}}\left(10^{-6}\right)$, \\
\hline 1 & 2.09 & $3 \mathrm{H}$ & 5 & 6.63 & $1 \mathrm{H}$ & 9 & 7.69 & $1 \mathrm{H}$ & $10.23 \quad 1 \mathrm{H}$ \\
\hline 2 & 2.14 & $3 \mathrm{H}$ & 6 & 6.64 & $1 \mathrm{H}$ & 10 & 7.89 & $1 \mathrm{H}$ & \\
\hline 3 & 3.83 & $3 \mathrm{H}$ & 7 & 7.41 & $1 \mathrm{H}$ & 11 & 8.16 & $1 \mathrm{H}$ & \\
\hline 4 & 3.88 & $3 \mathrm{H}$ & 8 & 7.69 & $1 \mathrm{H}$ & 12 & 9.84 & $1 \mathrm{H}$ & \\
\hline
\end{tabular}

Research on synthesis process of group-E-diphenylethene compounds (DSPDA). The catalyst has a great influence on the experiment, the effects of the type and content of the catalyst on the property of the product were discussed, base catalyst was used in condensation between carbonyl compounds and active hydrogen compounds, only few cases use acid catalysis. In this paper, reaction chooses base catalysis, DSPDA formed by the condensation between MNPDA and benzene compounds with aldehyde, The focus of the reaction is to choose the appropriate base catalysis,which has appropriate power to take methyl hydrogen atoms of MNPDA ${ }^{[8]}$. As following figure, then methylene anion attacks aldehyde group, through the steps of methylene anion 
generated controls the whole reaction. In the experiment, the effect of several kinds of catalyst as piperidine,morpholine, sodium methoxide was investigated.

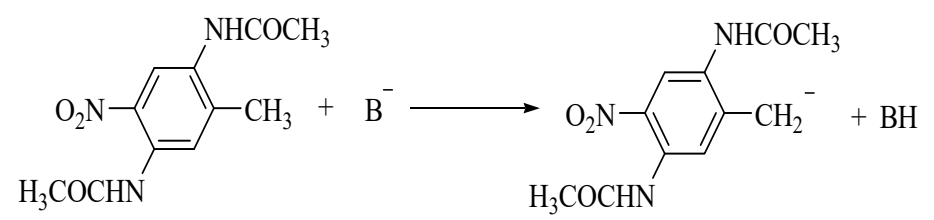

Figure 7.

Table 3. The influence of catalyst on reaction

\begin{tabular}{cccc}
\hline $\begin{array}{c}\text { Serial } \\
\text { number }\end{array}$ & Catalyst and its use & purity $(\%)$ & yield $(\%)$ \\
1 & piperidine $(2.0)$ & 90.67 & 68.65 \\
2 & piperidine $(2.5)$ & 93.14 & 73.20 \\
3 & piperidine (3.0) & 82.91 & 69.27 \\
4 & morpholine (4.0) & 73.12 & 49.88 \\
5 & morpholine (6.0) & 74.09 & 53.78 \\
6 & sodium methoxide (1.0) & $/$ & $/$ \\
7 & sodium hydroxide (1.0) $\mathrm{g}$ & $/$ & $/$ \\
8 & pyridine (3.0) & $/$ & $/$
\end{tabular}

Note: MNPDA2.51 g (0.01 mol) , DMBA3.15 g (0.02 mol), DMSO25 ml, molecular sieve powder $3 \mathrm{~g}$, temperature $130{ }^{\circ} \mathrm{C}$, reaction time, $11 \mathrm{~h}$

Using piperidine as catalyst, the reaction take smoothly, That means piperidine can just take a methyl hydrogen atoms of benzene into a methylene anion. Morphine's alkaline weaker than piperidine, No matter how much morphine used, The highest purity of target product is $74 \%$. In conclusion, piperidine was used as the catalyst, the purity and yield of target product reached highest when amount of piperidine is $25 \mathrm{ml}$.

Such as DMSO andDMF, the molecule's positive charge of polar aprotic solvent are dispersed, space block also is big, so anion solvation is difficult, polar aprotic solvent also difficult to react with electronegativity of nucleophilic reagent. On the other hand, Solvent cathode is generally on the oxygen atoms,cathode exposed on the surface of molecules and no space hampered, cathode set off ion dipole interaction with positive ions of the solute. It makes nucleophilic reagent activity increased. Reaction was easy to happen ${ }^{[10]}$. Experimental results also prove this view, it can be seen from table 4.

Table 4. solvent effects on DSPDA synthesis

\begin{tabular}{cccc}
\hline $\begin{array}{c}\text { Serial } \\
\text { number }\end{array}$ & $\begin{array}{c}\text { Solvent and its dosage } \\
(\mathrm{ml})\end{array}$ & $\begin{array}{c}\text { purity } \\
(\%)\end{array}$ & $\begin{array}{c}\text { yield } \\
(\%)\end{array}$ \\
\hline 1 & DMSO (25) & 93.14 & 73.20 \\
2 & Pyridine (25) & 45.78 & 25.34 \\
3 & DMF (25) & 71.00 & 38.56 \\
4 & Ethylene glycol (25) & $/$ & $/$ \\
\hline
\end{tabular}


Note: MNPDA $2.51 \mathrm{~g}(0.01 \mathrm{~mol})$, DMBA $3.15 \mathrm{~g}$ (0.02 mol), piperidine $2.5 \mathrm{ml}$, molecular sieve powder $3 \mathrm{~g}$, temperature $130{ }^{\circ} \mathrm{C}$, reaction time, $11 \mathrm{~h}$

\section{Discussion}

MNPDA and DMBA as raw material, DMSO as the solvent, piperidine as catalyst, molecular sieve powder as dehydration agent, the innovative was synthesizing new optical materials monomer DSPDA by condensation, experimental condition optimization was as follows: DMSO as solvent, molecular sieve powder as dehydration agent, reaction temperature was $130{ }^{\circ} \mathrm{C}$, the ratio of raw materials is $\mathrm{n}(\mathrm{MBA}): \mathrm{n}$ (MNPA) $=2: 1$, reaction time is 11 hours and amount of piperidine was 2.5 ml. The purity of MSPDA was $93.14 \%$ and the yield was $73.20 \%$. By means of FT-IR, ${ }^{1} \mathrm{H}-\mathrm{NMR}$ and ${ }^{13} \mathrm{C}$-NMR determination and qualitative analysis, to confirm the $N, N$-diacetyl-2-(2,4dimethoxystyryl)-5-nitro-1,4-phenylenediamine(DSPDA).

Not join molecular sieve in the reaction, this process is a better choice for industrialized production, get the product purity was $86.88 \%$, yield was $82.66 \%$. Product purity can reach $99 \%$ after purified, This method is suitable for industrial production.

\section{References}

[1] Franken P A, Hill A E, Peters C W, et al.Generation of optical harmonics[J]. Phys Rev Lett,1961,7(4):118-119.

[2] LIU Guan xin, XI Hong xia, WEI Li fei, et al. Synthesis of neworganic/inorganic hybrid nonlinear optical material[J]. Journal of Optoelectronics 'Laser, 2004, 15(4) : 4592462. (in Chinese).

[3] XIE Hong quan, LIU Zhi hong, HUANG Xu dong, et al. Synthesis and nonlinear optical properties of four polyurethanes containing different chromophore groups[J]. European Polymer Journal, 2001, 37: 497-505.

[4] W. J. FARRISSEJYR., F. P. RECCHI. Condensation of p-Nitrotoluene with Aldehydes[J]. The Journal of Organic Chemistry, 1969, 34: 2785-2786.

[5] Damore F, Lanata M., Pietralunga S., et al. Enhancement of PMMA nonlinear optical properties by means of a quinoid molecule[J]. OPtical Materials, 2004, 24(4): 661-665.

[6] Shashi P. Karna. Electronic and Nonlinear Optical Materials: The Role of Theory and Modeling[J]. J. Phys. Chem. A, 2000, 104(20), 4671-4673.

[7] Weder, C.; Neuenschwander, P.; Suter,U.W.; Pretre, P.; Kaatz, P.; Gunter, P. New Poly- amides with Large Second-Order Nonlinear Optical Properties[J]. Macromolecules, 1994, 27: 2181.

[8] Kim, T. D. Lee, K. S. Lee, G. U. Kim, O. K. Synthesis and characterization of a novel polyimide-based second-order nonlinear optical material[J]. Polymer, 2000, 41 (14): 5237-5245.

[9]Shanyi Guang, Shouchun Yin. Synthesis and properties of long conjugated organic optical limiting materials with different $\pi$-electron conjugation bridge structure[J]. Dyes and Pigments, 2007, 73: 285-291. 